\title{
Impact of thoracic paravertebral block combined with general anesthesia on postoperative cognitive function and serum adiponectin levels in elderly patients undergoing lobectomy
}

\author{
Haihui Xie ${ }^{1}$, Jianping Zhou², Wei Du², Shu Zhang ${ }^{1}$, Runcheng Huang ${ }^{1}$, Qi Han ${ }^{1}$, Qingcong Guo ${ }^{1}$ \\ ${ }^{1}$ Department of Anesthesiology, Affiliated Dongguan People's Hospital of Southern Medical University, Dongguan, China \\ ${ }^{2}$ Department of Thoracic Surgery, Affiliated Dongguan People's Hospital of Southern Medical University, Dongguan, China
}

Videosurgery Miniinv 2019; 14 (4): 538-544

DOI: https://doi.org/10.5114/wiitm.2019.84742

\begin{abstract}
Introduction: Thoracic paravertebral block (TPVB) can improve postoperative analgesia, inhibiting perioperative stress and the inflammatory response. However, whether it can improve early postoperative cognitive dysfunction $(P O C D)$ by altering the secretion and expression of serum ADP remains unclear.

Aim: To investigate the impact of thoracic paravertebral block combined with general anesthesia (TPVB-GA) on postoperative cognitive function and serum adiponectin (ADP) levels in elderly patients undergoing lobectomy.

Material and methods: A total of 120 elderly patients undergoing elective lobectomy were randomly divided into three groups: general anesthesia (GA); TPVB-GA (PG); epidural block combined with general anesthesia (EG). Cognitive function in the three groups was evaluated 1 day before and 7 days after surgery. The serum levels of ADP and $S-100 \beta$ protein were evaluated before anesthesia (TO), 15 min after skin incision (T3), and 7 days after surgery (T5). Results: Cognitive function scores in the three groups decreased by different extents at $T 5(p<0.05)$; scores in groups $P G$ and $E G$ were higher than those in group $G A(p<0.05)$. The serum levels of $S-100 \beta$ protein in the three groups at $T 3$ were higher than those at TO $(p<0.05)$; however, serum ADP concentrations were reduced $(p<0.05)$; the serum levels of $S-100 \beta$ protein in groups $P G$ and $E G$ were lower than those in group GA at T3, while serum ADP levels were higher.

Conclusions: Thoracic paravertebral block or epidural block combined with general anesthesia can improve early postoperative cognitive function in elderly patients undergoing lobectomy. TPVB-GA demonstrated better effects, which may be related to the secretion of ADP.
\end{abstract}

Key words: thoracic paravertebral block, lobectomy, postoperative cognitive dysfunction, adiponectin, elderly.

\section{Introduction}

Thoracic surgery is characterized by extensive trauma, stress response, postoperative pain, and severe systemic inflammatory reactions, which are prone to cause a variety of perioperative complications, especially in elderly patients due to their often frail nature and degenerated physiological function. Postoperative cognitive dysfunction (POCD) is a common complication of thoracic surgery in elderly patients, with a reported incidence of $13 \%$ to $47 \%$ [1]. The incidence of POCD is closely related to the method and management of surgical anesthesia, as well as to the stress response and the release of inflammatory mediators caused by anesthetics $[2,3]$. Appropriate anesthetic management can reduce perioperative stress and inflammatory reac-

\section{Address for correspondence}

Jianping Zhou, Department of Thoracic Surgery, Affiliated Dongguan People's Hospital of Southern Medical University, Dongguan, China, e-mail: cnjianpingzhou@126.com 
tions, reduce perioperative complications, and improve patient prognosis. Adiponectin (ADP) is a fat cell-derived hormone protein secreted by adipose tissue. It has several properties, including anti-inflammatory, antioxidant, and glucose-lowering effects, which in turn confer protective effects on cells, tissues, and organs [4-6]. Studies have reported that ADP can effectively reduce the secretion of central pro-inflammatory factors, protect against cognitive dysfunction, and delay the progression of cognitive impairment [7]. In previous studies, we found that postoperative serum ADP levels in elderly patients undergoing general anesthesia were negatively correlated with the occurrence of $\mathrm{POCD}[8,9]$.

Thoracic paravertebral block (TPVB) can directly block nerve conduction on the operative side, which helps to improve the analgesic effect and, because, it only blocks the paravertebral nerve, it has little effect on physiological function in the rest of the body [10]. There have been many reports describing its impact on perioperative stress, inflammatory reactions, and postoperative analgesia. However, whether it can improve early POCD by altering the secretion and expression of serum ADP remains unclear.

\section{Aim}

The purpose of this study was to evaluate the impact of TPVB-GA on postoperative cognitive function and serum ADP level in elderly patients undergoing thoracoscopic lobectomy, and to provide a reference for the selection of appropriate clinical anesthesia in such procedures.

\section{Material and methods}

\section{General information}

This study was approved by the Medical Ethics Committee of Dongguan People's Hospital (Lunshen 2017-77); the patients or their guardians provided informed written consent. A total of 120 elderly patients undergoing thoracoscopic lobectomy in the authors' hospital were selected. Inclusion criteria were as follows: age 65-81 years; undergoing elective thoracoscopic lobectomy; American Society of Anesthesiologists grade II or III; and New York Heart Association grade I-II cardiac function. Exclusion criteria were as follows: preoperative Mini-Mental State Examination (MMSE) score $<26$ points (1 day before surgery); unable to recognize text in the cognitive function test table; significant respiratory or circulatory system disease, hepatonephric dysfunction, or mental and/or neurological disease before surgery; history of preoperative radiotherapy or chemotherapy, or abnormal coagulation function; antipsychotic or sedative medications, or narcotic analgesics within 2 years of the surgery; history of alcohol abuse or drug dependence; severe vision or hearing impairment or other factors that may result in non-communication with the reviewers; intraoperative blood loss $>800 \mathrm{ml}$; or surgery time $>3 \mathrm{~h}$. The patients were randomly divided into three groups using a randomized digital table method, with 40 patients per group: general anesthesia (GA); TPVB-GA (PG); and epidural block combined with general anesthesia (EG).

\section{Anesthesia}

Each patient was routinely monitored for invasive blood pressure (BP), oxygen saturation $\left(\mathrm{SpO}_{2}\right)$, electrocardiogram (ECG), and Nactrend depth of anesthesia after entering the operating room, together with establishing intravenous access and undergoing right internal jugular vein catheterization under local anesthesia. All patients underwent dual-lumen bronchial catheterization for general anesthesia, and arterial BP was continuously monitored using transradial catheterization. Group PG underwent ultrasound-guided TPVB [11] before anesthesia induction (Venue50-type ultrasound system, GE Healthcare, USA) for positioning and guidance. The method of T4 point-block and catheterization was selected, followed by injection of $20 \mathrm{ml}$ of $0.25 \%$ ropivacaine to determine the block plane for the induction of anesthesia. Group EG underwent epidural block before the induction of anesthesia. After successful puncture at T6-7, $3 \mathrm{ml}$ of $2 \%$ lidocaine (initial dose) was administered and, after confirming that the catheter was located in the epidural space, $8-15 \mathrm{ml}$ of $0.375 \%$ ropivacaine was intermittently injected, followed by confirming the block plane and starting induction of anesthesia. Group GA underwent direct induction of anesthesia, which involved intravenous injection of midazolam ( 0.05 to $0.1 \mathrm{mg} / \mathrm{kg}$ ), etomidate $(0.3 \mathrm{mg} / \mathrm{kg})$, sufentanil $(0.4 \mu \mathrm{g} / \mathrm{kg})$, and rocuronium $0.6 \mathrm{mg} / \mathrm{kg}$. After oral dual-lumen endobronchial catheterization and confirming correct positioning of fiberoptic bronchoscopy, volume-controlled ventilation was performed, with a tidal volume of $6-8 \mathrm{ml} / \mathrm{kg}$, ventilation frequency 
15 breaths/min, inhalation ratio $1: 2$, and end-tidal carbon dioxide maintained at 30-35 $\mathrm{mm} \mathrm{Hg}$. Intraoperative anesthesia was maintained by constant infusion of propofol, remifentanil, and cisatracurium so as to maintain the Narcotrend index at D2-E1. Body temperature was also monitored and maintained at $36^{\circ} \mathrm{C}$ to $37^{\circ} \mathrm{C}$ intraoperatively. Intraoperative urine volume, blood loss, and infusion/transfusion volume were also recorded. All groups were administered postoperative intravenous patient-controlled analgesia ( $\mathrm{PCIA})$, and analgesic drugs were prepared as follows: $100 \mu \mathrm{g}$ of sufentanil $+200 \mathrm{mg}$ of flurbiprofen axetil $+8 \mathrm{mg}$ of tropisetron (diluted to $200 \mathrm{ml}$ with saline). The background infusion rate was $2 \mathrm{ml} / \mathrm{h}$, together with a PCIA dose of $2 \mathrm{ml}$ with a lock-out time of $15 \mathrm{~min}$. Each patient assessed the degree of pain using a postoperative visual analog scale (VAS) [12] (0 = no pain, $10=$ severe pain) and maintained a VAS score $\leq 3$ points.

\section{Hemodynamic monitoring}

The mean arterial pressure (MAP) and heart rate $(\mathrm{HR})$ of each patient were measured at different time points: before anesthesia (T0); 15 min after paravertebral or epidural anesthesia (T1); at intubation (T2); 15 min after incision (T3); and pre-extubation (T4).

\section{Assessment of cognitive function}

The MMSE, digital symbol test, trail connection test $A$, word recognition test, and digital breadth test (forward and reverse recitation) [13] were performed 1 day before and 7 days after surgery by a researcher blinded to the grouping allocation to assess cognitive function of the patients in the three groups. The standard deviation of all test scores for each patient before surgery was calculated and compared with scores after surgery. Patients with a mean decrease in score $\geq 1$ standard deviation were considered to have experienced cognitive dysfunction after surgery. Patients who exhibited cognitive dysfunction in $\geq 2$ tests were considered to have POCD [14].

\section{ELISA}

Venous blood ( $3 \mathrm{ml}$ ) was collected at T0, T3, and 7 days after surgery (T5) to assess serum ADP (BL OTANG Inc, Lexington MA, USA) and S-100 $\beta$ protein (BL OTANG Inc, Lexington MA, USA) levels using ELISA.

\section{Statistical analysis}

SPSS version 17.0 (IBM Corporation, Chicago, IL, USA) was used for statistical analysis. Measurement data are expressed as mean \pm standard deviation. The intragroup comparison used single-factor analysis of variance or multivariate analysis of variance. Inter-group comparisons were performed using the group t-test, and measurement data were analyzed using the $\chi^{2}$ test, with $p<0.05$ considered to be statistically significant.

\section{Results}

\section{Generation information}

Six patients included in this study were lost to follow-up after surgery (1 patient in group GA, 2 in group $P G$, and 2 in group EG), and 2 patients who developed complications and were transferred to the intensive care unit after treatment (1 patient in group PG and 2 patients in group EG). A total of 112 patients completed the study.

There was no significant difference in age, body mass index, anesthesia time, operation time, or intraoperative blood loss among the three groups $(p>0.05)$ (Table I).

\section{Perioperative hemodynamic changes}

There were no data for group GA at TO because epidural or paravertebral block was not performed. Comparing data at TO, MAP and HR in group GA were significantly increased from T2 to T4 $(p<0.05)$, and MAP in group EG was significantly decreased from T1 to T4 $(p<0.05)$; however, HR was signifi-

Table I. Comparison of general data among the three groups $(\bar{x} \pm s)$

\begin{tabular}{|lcccc|}
\hline Group & Age [years] & BMI $\left[\mathrm{kg} / \mathrm{m}^{2}\right]$ & $\begin{array}{c}\text { Anesthesia time } \\
{[\mathrm{min}]}\end{array}$ & $\begin{array}{c}\text { Operation time } \\
{[\mathrm{min}]}\end{array}$ \\
\hline GA & $76.63 \pm 4.60$ & $22.71 \pm 2.8$ & $103.6 \pm 16.8$ & $83.6 \pm 13.6$ \\
\hline PG & $75.13 \pm 5.60$ & $22.69 \pm 3.2$ & $104.3 \pm 15.6$ & $83.0 \pm 14.8$ \\
\hline EG & $76.13 \pm 5.60$ & $22.66 \pm 4.06$ & $102.8 \pm 17.0$ & $82.96 \pm 14.38$ \\
\hline
\end{tabular}


cantly decreased from T1 to T3 $(p<0.05)$. MAP and $H R$ in groups $E G$ and $P G$ were lower than those in group GA from T2 to T4 $(p<0.05)$, and MAP and HR in group PG were higher than those in group $E G$ from T1 to T3 $(p<0.05)$ (Table II).

\section{Cognitive function score}

There was no significant difference in cognitive function score among the three groups 1 day before surgery $(p<0.05)$. Comparing data collected 1 day before surgery, scores from the MMSE, digital symbol test, digital breadth test, and trail connection test $A$ in the three groups 7 days after surgery were reduced $(p<0.05)$, but there was no significant difference in scores on the word recognition test among the three groups $(p>0.05)$. Intergroup comparison of the scores from the MMSE, digital symbol test, digital breadth test, and trail connection test $A$ in group GA were lower than in groups PG and EG 7 days after surgery $(p<0.05)$. These scores in group PG were higher than those in group EG (Table III).

\section{Incidence of POCD 7 days after surgery}

The incidence of POCD in group GA was 18\% (7 patients), $7.7 \%$ in group PG (3 patients), and $12.8 \%$ in group EG (5 patients), with differences that were statistically significant $(p<0.05)$.

\section{Serum ADP and S-100 $\beta$ protein levels}

Serum ADP levels decreased in the three groups at T3 and T5 compared with T0 $(p<0.05)$; however, serum ADP levels in groups PG and EG were higher than those in group GA at T3 and T5 $(p<0.05)$. Serum ADP level in group PG was higher than that in group EG at T5. Serum S-100 $\beta$ protein levels were increased in the three groups at T3 and T5 compared with T0 $(p<0.05)$; however, serum S-100 $\beta$ protein levels in groups PG and EG were lower than those in group GA at T3 and T5 $(p<0.05)$. Serum S- $100 \beta$ protein level in group PG was lower than that in group EG at T5 (Table IV).

\section{Discussion}

Thoracic surgery is often associated with many perioperative complications and is currently considered to be associated with severe perioperative stress reactions that reduce physiological reserve capacity, cause cytokine imbalance, and increase the inflammatory response [15]. Appropriate analgesia can reduce pain caused by surgical trauma, which in turn reduces the inflammatory response [16], which is of great significance in reducing perioperative complications and promoting postoperative rehabilitation. Recent studies have reported that local anesthesia can reduce the surgical stress response [17]. Epidural block can adequately control pain; however, its applications are limited due to its impact on perioperative hemodynamics and urinary retention. Recently, ultrasound-guided thoracic paravertebral block combined with general anesthesia has gradually replaced epidural anesthesia for thoracic surgery because of its simplicity, safety, effectiveness, and fewer complications, and is especially suitable for some patients in whom epidural block is difficult. The results of this study demonstrated that perioperative MAP and HR were significantly increased in group GA, perioperative MAP was significantly decreased in group EG, and HR was significantly diminished. Perioperative MAP and HR exhibited no significant change in group PG, indicating that TPVB-GA had a smaller impact on perioperative hemodynamics. TPVB blocks the body and the sympathetic nerves on the operative side, and inhibits the body's

Table II. Comparison of hemodynamic changes among the three groups $(\bar{x} \pm s, n=60)$

\begin{tabular}{|c|c|c|c|c|c|c|c|}
\hline Group & $N$ & Index & T0 & $\mathrm{T} 1$ & $\mathrm{~T} 2$ & T3 & T4 \\
\hline \multirow[t]{2}{*}{ GA } & 39 & MAP [mm Hg] & $92 \pm 16$ & - & $107 \pm 16^{a}$ & $106 \pm 18^{a}$ & $99 \pm 10^{a}$ \\
\hline & & HR [beats/min] & $75 \pm 13$ & - & $86 \pm 9^{a}$ & $85 \pm 8^{a}$ & $83 \pm 10^{a}$ \\
\hline \multirow[t]{2}{*}{$P G$} & 37 & MAP [mm Hg] & $93 \pm 13$ & $92 \pm 11^{c}$ & $92 \pm 16^{b c}$ & $93 \pm 11^{b c}$ & $91 \pm 16^{b c}$ \\
\hline & & HR [beats/min] & $74 \pm 16$ & $73 \pm 12^{c}$ & $76 \pm 16^{b c}$ & $76 \pm 13^{b c}$ & $73 \pm 12^{b}$ \\
\hline \multirow[t]{2}{*}{$E G$} & 36 & $\mathrm{MAP}[\mathrm{mm} \mathrm{Hg}]$ & $92 \pm 11$ & $78 \pm 13^{a}$ & $76 \pm 12^{\mathrm{ab}}$ & $86 \pm 13^{a b}$ & $87 \pm 16^{a b}$ \\
\hline & & $\mathrm{HR}$ [beats/min] & $75 \pm 18$ & $66 \pm 12^{a}$ & $63 \pm 12^{a b}$ & $66 \pm 10^{a b}$ & $73 \pm 16^{b}$ \\
\hline
\end{tabular}

Compared with the data at T0, ${ }^{a} p<0.05$; compared with group $G A,{ }^{b} p<0.05$; compared with group $E G,{ }^{c} p<0.05$. 
Table III. Comparison of cognitive function changes before and after surgery among the 3 groups $(\bar{x} \pm s)$

\begin{tabular}{|c|c|c|c|c|}
\hline Index & Group & $N$ & 1 day before surgery & 7 days after surgery \\
\hline \multirow[t]{3}{*}{ MMSE } & GA & 39 & $28.6 \pm 1.8$ & $26.1 \pm 1.6^{b}$ \\
\hline & $P G$ & 37 & $28.8 \pm 1.6$ & $27.9 \pm 1.8^{\mathrm{ab}}$ \\
\hline & $\mathrm{EG}$ & 36 & $28.7 \pm 1.9$ & $27.0 \pm 1.3^{\mathrm{ab}}$ \\
\hline \multirow[t]{3}{*}{ Digital symbol test } & GA & 39 & $12.8 \pm 1.9$ & $9.3 \pm 1.8^{b}$ \\
\hline & PG & 37 & $12.9 \pm 1.6$ & $11.9 \pm 1.6^{\mathrm{abc}}$ \\
\hline & EG & 36 & $12.9 \pm 1.0$ & $10.8 \pm 1.9^{a b}$ \\
\hline \multirow[t]{3}{*}{ Trail connection test $\mathrm{A}$} & GA & 39 & $23 \pm 13$ & $17 \pm 10^{b}$ \\
\hline & $P G$ & 37 & $24 \pm 10$ & $22 \pm 13^{a b c}$ \\
\hline & EG & 36 & $24 \pm 16$ & $20 \pm 12^{a b}$ \\
\hline \multirow[t]{3}{*}{ Word recognizing test } & GA & 39 & $3.8 \pm 1.3$ & $3.6 \pm 1.8$ \\
\hline & $P G$ & 37 & $3.6 \pm 1.6$ & $3.8 \pm 1.6$ \\
\hline & EG & 36 & $3.7 \pm 1.3$ & $3.6 \pm 1.7$ \\
\hline \multirow{3}{*}{$\begin{array}{l}\text { Digital breadth test } \\
\text { (forward recitation) }\end{array}$} & GA & 39 & $8.8 \pm 1.6$ & $6.0 \pm 1.8^{b}$ \\
\hline & $P G$ & 37 & $8.9 \pm 1.3$ & $7.9 \pm 1.6^{\mathrm{abc}}$ \\
\hline & $E G$ & 36 & $8.7 \pm 1.5$ & $7.1 \pm 1.7^{\mathrm{ab}}$ \\
\hline \multirow{3}{*}{$\begin{array}{l}\text { Digital breadth test } \\
\text { (reverse recitation) }\end{array}$} & GA & 39 & $4.7 \pm 1.3$ & $2.9 \pm 1.8^{b}$ \\
\hline & $P G$ & 37 & $4.8 \pm 1.6$ & $4.0 \pm 1.8^{\mathrm{abc}}$ \\
\hline & EG & 36 & $4.7 \pm 1.6$ & $3.6 \pm 1.8^{\mathrm{ab}}$ \\
\hline
\end{tabular}

Compared with group $G A,{ }^{a} p<0.05$; compared with the data 1 day before surgery, ${ }^{b} p<0.05$; compared with group $E G,{ }^{c} p<0.05$.

Table IV. Comparison of serum ADP and S-100 $\beta$ protein levels at different time points among the 3 groups

\begin{tabular}{|lcccccc|}
\hline Time point & \multicolumn{3}{c}{ ADP $[\mathrm{ng} / \mathrm{l}]$} & \multicolumn{3}{c|}{ S-100 $\beta$ protein [ng/l] } \\
\cline { 2 - 7 } & GA & PG & EG & GA & PG & EG \\
\hline T0 & $9.6 \pm 1.3$ & $9.5 \pm 1.6$ & $9.3 \pm 1.8$ & $127 \pm 16$ & $126 \pm 17$ & $123 \pm 18$ \\
\hline T3 & $5.7 \pm 1.6^{\mathrm{a}}$ & $7.9 \pm 1.3^{\mathrm{ab}}$ & $8.1 \pm 1.2^{\mathrm{ab}}$ & $226 \pm 17^{\mathrm{a}}$ & $173 \pm 15^{\mathrm{ab}}$ & $178 \pm 15^{\mathrm{ab}}$ \\
\hline T5 & $7.8 \pm 1.8^{\mathrm{a}}$ & $9.1 \pm 1.7^{\mathrm{abc}}$ & $8.2 \pm 1.6^{\mathrm{ab}}$ & $168 \pm 13^{\mathrm{a}}$ & $133 \pm 18^{\mathrm{abc}}$ & $156 \pm 15^{\mathrm{ab}}$ \\
\hline
\end{tabular}

Compared with $T 0,{ }^{a} p<0.05$; compared with group $G A,{ }^{b} p<0.05$; compared with group $E G,{ }^{c} p<0.05$.

stress response to surgical stimuli. At the same time, it does not interfere with heart function, has only a slight physiological impact on the body, and can maintain hemodynamic stability.

The POCD is a common complication after openchest procedures in elderly patients. Currently, there is no uniform standard for the diagnosis of POCD, and assessment is based primarily on neuropsychological tests. This study used 6 cognitive function scales for evaluation, which covered three aspects of cognitive function (except for language) but, nevertheless, accurately reflected cognitive function.
The MMSE is a commonly used method to evaluate cognitive function; the digital symbol test is used to evaluate attention and response speed; the digital breadth test is used to evaluate attention and short-term memory; the trail connection test $A$ is used to evaluate the information processing ability; and the word recognition test is used to evaluate long-term memory. The results of this study demonstrated that in the comparison between group PG and group EG, the scores from the MMSE, digital symbol test, digital breadth test and trail connection test $A$ in group GA decreased 7 days after surgery, 
indicating that compared with general anesthesia, TPVB or epidural block can significantly reduce postoperative cognitive impairment in elderly patients undergoing thoracotomy. There was no difference in word recognition test scores among the three groups, indicating that anesthesia had no effect on long-term memory.

The ADP is an adipocytokine with anti-inflammatory properties. It is abundant in human plasma, and its expression and secretion are regulated by inflammatory factors, reactive oxygen species, transcription factors, and/or hormones, such as tumor necrosis factor- $\alpha$, interleukin- 6 , or other inflammatory mediators that can inhibit the expression and secretion of ADP. In recent years, it has been found that the expression of and receptors for ADP are widely present in the pituitary and brain. Moreover, evidence that AdipoR1 exists in the hypothalamus and basal ganglia suggests that ADP may be involved in the central nervous system signaling pathway to control energy balance and higher brain function [18]. De Franciscis et al. [19] found that a significant positive association may exist between serum ADP levels and better cognitive function in postmenopausal women. The change in serum S-100 $\beta$ protein level is a specific indicator reflecting brain injury and cognitive functional changes [20]. The results of this study demonstrated that serum $\mathrm{S}-100 \beta$ protein levels in groups PG and EG were significantly lower than those in group GA at T3 and T5, but serum ADP levels in groups PG and EG were significantly lower than those in group GA. The incidence of POCD in groups $P G$ and EG was significantly lower than that in group GA 7 days after surgery, indicating that compared with simple general anesthesia, TPVB-GA or epidural block combined with GA can significantly reduce brain injury and inhibit the inflammatory response in elderly patients undergoing thoracotomy, and can reduce the incidence of POCD 7 days later.

A possible mechanism may be that simple general anesthesia cannot effectively inhibit the stress response of patients undergoing thoracoscopic surgery; therefore, such patients are prone to experiencing severe hemodynamic fluctuations, resulting in inflammatory reactions, increased secretion of inflammatory cytokines, and inhibited expression and secretion of ADP. Furthermore, the intraoperative use of anesthetics varies, especially opioid drugs, which can cause memory and cognitive dysfunction [21]. Epidural anesthesia can block sympathetic nerves in the corresponding segments, block the afferent of nocuous stimuli, reduce the release of catecholamines, and inhibit the stress response caused by surgical trauma. Meanwhile, it also has a direct inhibitory effect on the cardiac sympathetic nerves, thus causing severe intraoperative hypotension and bradycardia, resulting in intraoperative hemodynamic instability. TPVB, however, blocks only the somatosensory nerve of the operative side and has little effect on the body's normal physiology; therefore, its block of the sympathetic nerve that conducts pain is more complete than epidural block, it can effectively inhibit the harmful stress and inflammatory response to maintain stability of the internal environment [22]. Therefore, it can reduce inhibition of expression and secretion of ADP, which may be one of the important mechanisms of such anesthesia in improving postoperative cognitive dysfunction.

\section{Conclusions}

The TPVB or epidural block combined with general anesthesia can improve early postoperative cognitive function in elderly patients undergoing thoracoscopic surgery. The TPVB combined with general anesthesia is more effective and may be associated with the expression and secretion of ADP.

\section{Acknowledgments}

This study was funded by the Key Projects of Social Science and Technology Development (Dongguan, Guangdong, 201750715001459); Medical Research Foundation Projects of Guangdong (C2016044).

\section{Conflict of interest}

The authors declare no conflict of interest.

\section{References}

1. Price CC, Garvan CW, Monk TG. Type and severity of cognitive decline in older adults after noncardiac surgery. Anesthesiology 2008; 108: 8-17.

2. Lu X, Jin X, Yang S, Xia Y. The correlation of the depth of anesthesia and postoperative cognitive impairment: a meta-analysis based on randomized controlled trials. J Clin Anesth 2018; 45: 55-9.

3. Tang Y, Ouyang W. Inflammation-relevant mechanisms for postoperative cognitive dysfunction and the preventive strategy. Zhong Nan Da Xue Xue Bao Yi Xue Ban 2017; 42: 1321-6.

4. Sun Y, Zhao D, Yang Y, et al. Adiponectin exerts cardioprotection against ischemia/reperfusion injury partially via calreticulin 
mediated anti-apoptotic and anti-oxidative actions. Apoptosis 2017; 22: 108-17.

5. Kargulewicz A, Szulińska M, Kujawska-Łuczak M, et al. Improvement of serum adiponectin and leptin concentrations: effects of a low-calorie or isocaloric diet combined with metformin or orlistat - a prospective randomized open-label trial. Eur Rev Med Pharmacol Sci 2016; 20: 3868-76.

6. Han X, Wu Y, Liu X, et al. Adiponectin improves coronary no-reflow injury by protecting the endothelium in rats with type 2 diabetes mellitus. Biosci Rep 2017; 37: BSR20170282.

7. Zhang Y, Wang XL, Zhao J, et al. Adiponectin inhibits oxidative/nitrative stress during myocardial ischemia and reperfusion via PKA signaling. Am J Physiol Endocrinol Metab 2013; 305: E1436-43.

8. Xie H, Huang D, Zhang S, et al. Relationships between matrix metalloproteinase-9 (MMP-9) serum levels and postoperative cognitive dysfunction in elderly patients after general anesthesia. Aging Clin Exp Res 2016; 28: 1075-9.

9. Xie H, Zhang S, Huang R, et al. Impact of anesthesia depth on serum adiponectin, matrix metalloprotein 9 (MMP-9), and postoperative cognitive impairment in elderly patients with general anesthesia. Biomed Res 2017; 28: 5316-21.

10. Singh S, Jacob M, Hasnain S, Krishnakumar M. Comparison between continuous thoracic epidural block and continuous thoracic paravertebral block in the management of thoracic trauma. Med J Armed Forces India 2017; 73: 146-51.

11. Krediet AC, Moayeri N, van Geffen GJ, et al. Different approaches to ultrasound-guided thoracic paravertebral block: an illustrated review. Anesthesiology 2015; 123: 459-74.

12. Devesa JM, Vicente R, Abraira V. Visual analogue scales for grading faecal incontinence and quality of life: their relationship with the Jorge-Wexner score and Rockwood scale. Tech Coloproctol 2013; 17: 67-71.

13. Silbert B, Evered L, Scott DA, et al. Preexisting cognitive impairment is associated with postoperative cognitive dysfunction after hip jint replacement surgery. Anesthesiology 2015; 122: 1224-34.

14. Steinman L. Modulation of postoperative cognitive decline via blockade of inflammatory cytokines outside the brain. Proc Natl Acad Sci USA 2010; 107: 20595-6.

15. Talbot RM, McCarthy KF, McCrory C. Central and systemic inflammatory responses to thoracotomy - potential implications for acute and chronic postsurgical pain. J Neuroimmunol 2015; 285: 147-9.

16. Zhu A, Shen L, Xu L, et al. Suppression of Wnt5a, but not Wnts, relieves chronic post-thoracotomy pain via anti-inflammatory modulation in rats. Biochem Biophys Res Commun 2017; 493: 474-80.

17. Chen YQ, Jin XJ, Liu ZF, Zhu MF. Effects of stellate ganglion block on cardiovascular reaction and heart rate variability in elderly patients during anesthesia induction and endotracheal intubation. J Clin Anesth 2015; 27: 140-5.

18. Psilopanagioti A, Papadaki H, Kranioti EF, et al. Expression of adiponectin and adiponectin receptors in human pituitary gland and brain. Neuroendocrinology 2009; 89: 38-47.

19. De Franciscis P, Barbieri M, Leo $S$, et al. Serum adiponectin levels are associated with worse cognitive function in postmenopausal women. PLoS One 2017; 12: e0186205.
20. Li YC, Xi CH, An YF, et al. Perioperative inflammatory response and protein S-100beta concentrations - relationship with post-operative cognitive dysfunction in elderly patients. Acta Anaesthesiol Scand 2012; 56: 595-600.

21. Brock C, Olesen SS, Olesen AE, et al. Opioid-induced bowel dysfunction: pathophysiology and management. Drugs 2012; 72: 1847-65.

22. Copik M, Bialka S, Daszkiewicz A, Misiolek H. Thoracic paravertebral block for postoperative pain management after renal surgery: a randomised controlled trial. Eur J Anaesthesiol 2017; 34: 596-601.

Received: 14.01.2019, accepted: 2.03.2019. 